EPJ Web of Conferences 43, 03011 (2013)

DOI: $10.1051 /$ epjconf/20134303011

(C) Owned by the authors, published by EDP Sciences, 2013

\title{
Red giants rotational splittings
}

\author{
R.M. Ouazzani ${ }^{1,2, a}$, M.J. Goupil ${ }^{2}$, M.A. Dupret $^{1}$ and J.P. Marques ${ }^{1,3}$ \\ ${ }^{1}$ Institut d'Astrophysique et de Géophysique de l'Université de Liège, Belgium \\ ${ }^{2}$ Observatoire de Paris, LESIA, CNRS UMR 8109, 92195 Meudon, France \\ ${ }^{3}$ Georg-August-Universität Göttingen, Germany
}

\begin{abstract}
The space missions CoRoT and Kepler provide high quality data that allow to test the transport of angular momentum in stars by the seismic determination of the internal rotation profile. Our aim is to test the validity of the seismic diagnostics for red giant rotation that are based on a perturbative method and to investigate the oscillation spectra when the validity does not hold. We use a non-perturbative approach implemented in the ACOR code [1] that accounts for the effect of rotation on pulsations, and solves the pulsations eigenproblem directly for dipolar oscillation modes. We find that the limit of the perturbation to first order can be expressed in terms of the core rotation and the period separation between consecutive dipolar modes. Above this limit, each family of modes with different azimuthal symmetry $m$, has to be considered separately. For rapidly rotating red giants, new seismic diagnostics can be found for rotation by exploiting the differences between the period spacings associated with each $m$-family of modes.
\end{abstract}

\section{INTRODUCTION}

The CoRoT [2] and Kepler [3] spacecrafts have dramatically improved the quality of the available asteroseismic data. Several recent studies reported the detection of mixed modes that are split by rotation in a subgiant [4] and in several red giants [5-7] observed with Kepler. A large number of these stars exhibit frequency spectra that show a quite simple structure where symmetric patterns around axisymmetric modes are easily identified. They are interpreted as multiplets of modes split by rotation. The rotational splitting, i.e. the frequency spacing related to the lift of degeneracy caused by rotation, is then used to determine the core rotation. The values of the corresponding splittings are quite small and the use of the lowest order approximation to derive the splittings from stellar models for comparison can be justified. Such studies led to the determination of unexpectedly low central rotation frequencies (of a few hundreds of $\mathrm{nHz}$ ). These results are in strong disagreement with the core rotation frequencies predicted by evolutionary models, which are of the order of a few dozens of $\mu \mathrm{Hz}[8,9]$. They show that the transport processes induced by rotation currently included in stellar models are not able to sufficiently spin down the core of slowly core rotating red giants. On the other hand, a large set of red giant stars show complex frequency spectra, in particular with non symmetric multiplets and therefore these stars are likely fast rotators. Their rotation must then be investigated with nonperturbative methods.

In this context, we first report on the relevance of using a first order approach for the inference of rotation from seismic spectra of red giant stars with slowly to rapidly rotating core. When not relevant, we adopt the non-perturbative approach in order to shed light on the behaviour of splitted mixed modes in red giants spectra.

\footnotetext{
ae-mail: rhita-maria.ouazzani@ulg.ac.be
}

This is an Open Access article distributed under the terms of the Creative Commons Attribution License 2.0, which permits unrestricted use, distribution, and reproduction in any medium, provided the original work is properly cited. 
Figure 1. Pulsation frequencies in the inertial frame $v$ (in $\mu \mathrm{Hz}$ ) versus core rotation frequency $\Omega_{c} / 2 \pi$ (in $\mu \mathrm{Hz}$ ) for dipolar multiplets. Solid lines refer to $\mathrm{m}=-1$ (prograde) modes, dotted lines to $\mathrm{m}=0$ modes (axis-symmetric), and dashed lines to $m=1$ (retrograde) modes, for central rotation frequency ranging from 0 to

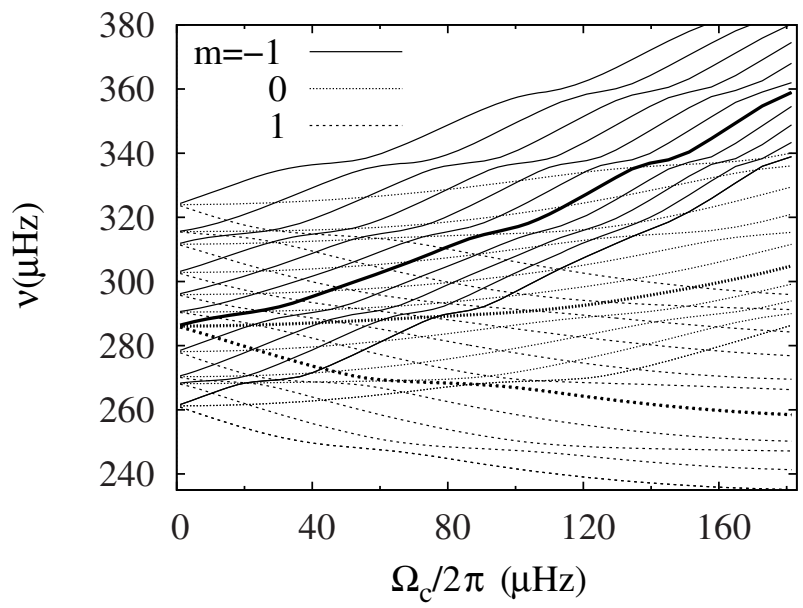
$100 \mu \mathrm{Hz}$.

\section{THEORETICAL FREQUENCY SPECTRA FOR ROTATING RED GIANTS}

This work is based on the study of a model (later on, model $\mathrm{M}_{1}$ ) at the bottom of the red giant branch (RGB), where the transport of angular momentum induced by rotation is included according to [10]. The stellar model is computed with the CESTAM code [11]. The central rotation rate predicted by the evolution model is $\Omega_{c} / 2 \pi \simeq 180 \mu \mathrm{Hz}$ while the surface convective region rotates at a rate of $1 \mu \mathrm{Hz}$. As noted by [11], the distortion of the model due to the centrifugal force can be neglected everywhere in red giant stars, and all the modes are mostly affected by the Coriolis force in the very inner layers of the star. In order to investigate the effect of core rotation on the frequency spectrum, we compute sets of frequencies for model $\mathrm{M}_{1}$ for a sequence of rotation profiles. This sequence is obtained arbitrarily by dividing the rotation profile given by CESTAM for $\mathrm{M}_{1}$ by constant factors. The oscillation frequencies are calculated by the non-perturbative pulsation code ACOR [1]. We also find out that two spherical harmonics $Y_{\ell}^{m}$ (for the scalar perturbed quantities, the radial and the poloidal perturbed velocity), and $Y_{\ell+1}^{m}$ (for the toroidal perturbed velocity) in the spectral expansion were sufficient. For the model $\mathbf{M}_{1}$, scaling laws [12] give a frequency of maximum power around $v_{\max }=289 \mu \mathrm{Hz}$ and a large separation of $\Delta v=23 \mu \mathrm{Hz}$. We then compute frequencies in the range $v_{\max } \pm 2 \Delta \nu$.

In Fig. 1 are presented the results of such calculations, i.e. the pulsation frequencies of several dipolar $(\ell=1)$ multiplets with increasing core rotation frequency. Starting at low rotation rate, due to the combined action of the Doppler effect and the Coriolis force, in the inertial frame, prograde $(m=-1)$ modes are shifted towards higher frequencies, whereas retrograde $(m=+1)$ modes are shifted towards lower frequencies. Frequencies of modes with different symmetry can cross each other when increasing the central rotation frequency. These crossings occur approximately for $\Omega_{c} / 2 \pi \gtrsim 8 \mu \mathrm{Hz}$ (for model $\mathrm{M}_{1}$ ). As a result, for frequencies above $8 \mu \mathrm{Hz}$, modes of different $m$ are no longer gathered by original multiplets in the frequency spectrum. By original multiplets, we mean triplets of modes which have the same degenerate frequency without rotation.

At this stage, it is important to recall that the trapping of modes essentially depends on their frequency (see for instance [13]). When $\Omega_{c}$ increases, so does the frequency difference between the three components of a triplet, and the trapping of these components can be significantly different, to such an extent that they end up with very different p-g nature. That is illustrated in Fig. 2, where the kinetic energy corresponding to modes of different $m$ around $290 \mu \mathrm{Hz}$ is plotted. It gives an indication of the p-g nature of the modes: the p-dominated modes (referred as p-m modes) lie in the minima of energy, while the g-dominated ones (g-m modes) are located in the maxima. The three modes circled in black belong to the same original multiplet, and show quite different $\mathrm{p}-\mathrm{g}$ nature. The prograde and the retrograde modes are g-m modes, while the $m=0$ one is a $\mathrm{p}-\mathrm{m}$ mode. This change of nature induced 


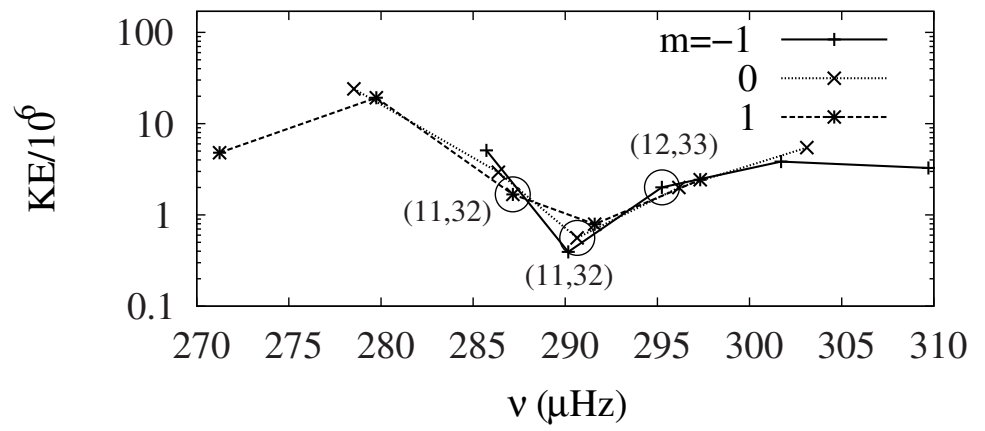

Figure 2. Kinetic energy of modes around a $\mathrm{p}-\mathrm{m}$ mode for model $\mathrm{M}_{1}$ centrally rotating at $20 \mu \mathrm{Hz}$. The three modes circled in black belong to the same original triplet. Inside the parentheses are indicated their number of nodes in the $\mathrm{p}$ cavity and in the g cavity.

by rotation, which depends on the azimuthal order, can also be characterized by looking at the number of nodes in the p-mode cavity $\left(\mathrm{n}_{\mathrm{p}}\right)$ and in the g-mode cavity $\left(\mathrm{n}_{\mathrm{g}}\right)$ (calculated according to the Cowling approximation, [14]). As shown in Fig. 2, the $n_{p}$ and $n_{g}$ values of the components of an original triplet are modified differently. These gains (for prograde and axi-symmetric modes) or losses (for retrograde modes) of nodes occur during avoided crossings. When increasing the rotation rate, if the frequencies of two modes of same symmetry become very close, they avoid to cross each other and exchange nature, as illustrated in the top part of Fig. 1. These avoided crossings need to be taken into account all the more because they mainly affect the p-m modes that are the most likely to be observed. Furthermore, the Coriolis force modifies the propagation cavities themselves (see [15], and references therein), and this change depends on the $m$ value of the mode. We expect this effect to play a role at moderate to high rotation velocity, and this will also impact the nature of modes.

Therefore, modes of different azimuthal order $m$, probe differently the stellar interior. Even if they belong to the same triplet with radial order $n$ and degree $\ell$, they can be of very different nature, and the use of the rotational splitting $\Delta_{n, \ell, m}$ defined as $\Delta_{n, \ell, m}=\left(\omega_{n, \ell, m}-\omega_{n, \ell,-m}\right) / 2 m$ to determine the rotation rate is therefore questionable for red giants.

\section{SLOW TO MODERATE CORE ROTATING RED GIANTS}

As illustrated in Fig. 1, for models with $\Omega_{c} / 2 \pi \leq 8 \mu \mathrm{Hz}$, the frequencies behave linearly with respect to $\Omega_{c}$. In this range, the sectorial modes $(\ell=|m|)$ are symmetrically distributed around the axi-symmetric modes, and have the same number of nodes in each cavity.

In this case, a first order perturbation approach approximates well the effects of rotation on the mode frequencies. The pulsation $\omega_{n, \ell, m}$ is then given by: $\omega_{n, \ell, m}=\omega_{n, \ell, 0}+m \Delta_{n, \ell}^{1}$ ([16] and [17]) where the rotational splitting $\Delta_{n, \ell}^{1}$ is expressed as a weighted measure of the star rotation rate:

$$
\Delta_{n, \ell}^{1}=\int_{0}^{R} K_{n, \ell}(r) \Omega(r) \mathrm{dr},
$$

where the functions $K_{n, \ell}(r)$ are the rotational kernels of the modes. They depend on the equilibrium structure and on the eigenfunctions of the unperturbed modes (see e.g. [18], and references therein). This formulation relies on two main assumptions. First, only the Coriolis force is significant and accounting for its contribution to order $O\left(\Omega / \omega_{n, \ell}\right)$ is sufficient. Second, Eq. (1) derives from a variational principle (see e.g. [19]), which requires that the eigenfunction of the mode perturbed by rotation is close to the eigenfunction of the unperturbed mode. This is the case for model $\mathrm{M}_{1}$ when the central rotation frequency is lower than $8 \mu \mathrm{Hz}$.

Between 8 and $20 \mu \mathrm{Hz}$ (Fig. 1), the mode frequencies seem to behave linearly with $\Omega_{c}$, but are no longer gathered by original multiplets. Figure 3 (top panel) displays the apparent rotational splittings, taking the half difference between the closest modes of opposite azimuthal order $m= \pm 1$. The values taken by the apparent rotational splittings range from few hundreds of $\mathrm{nHz}$ to $2 \mu \mathrm{Hz}$. This is of the order 
Figure 3. For the model $\mathrm{M}_{1}$ with a central rotation frequency of $20 \mu \mathrm{Hz}$, Top: spacing between two closest modes of opposite azimuthal order $\mathrm{m}= \pm 1$, i.e. apparent rotational splitting. Middle: Integral expressed in Eq. 1 where the kernel is associated to the each $m$-components of a triplet. Solid lines refer to $m=-1$ (prograde) modes, dotted lines to $\mathrm{m}=0$ modes (axis-symmetric), and dashed lines to $\mathrm{m}=1$ (retrograde) modes. Bottom: spacing between prograde and the retrograde modes which belong to the same original multiplet.

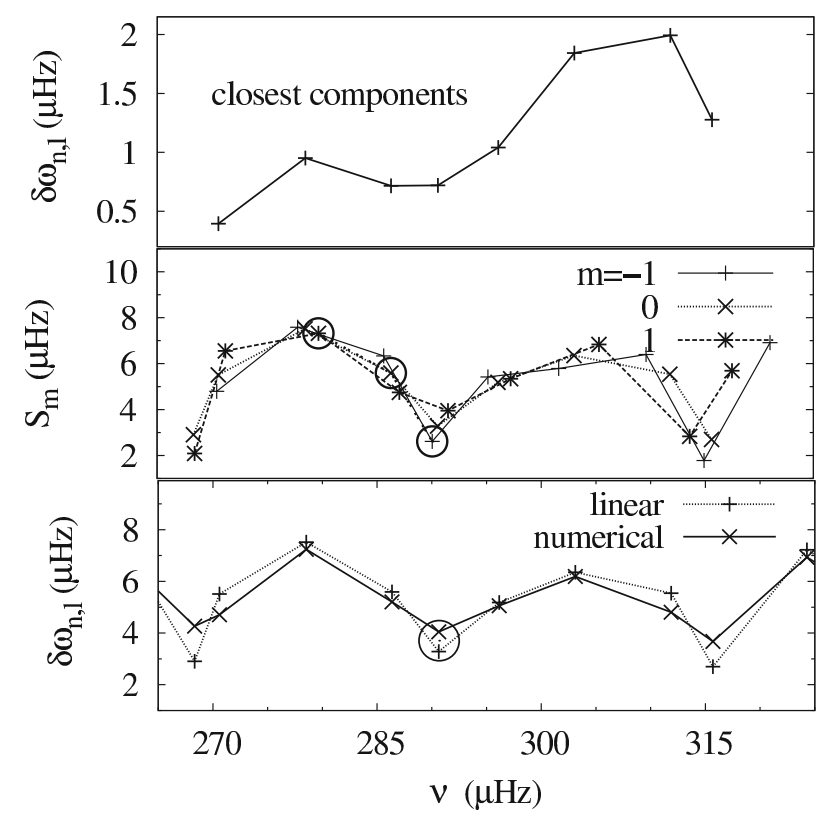

of the splittings measured in observed spectra. Because the apparent multiplets occur by 'accident', the curve in Fig. 3 (top panel) does not- and usually cannot- follow the particular ' $\mathrm{V}$ ' pattern around the $\mathrm{p}-\mathrm{m}$ modes as observed for some slowly rotating red giants (see for observations $[5,6]$ ). This ' $\mathrm{V}$ ' pattern is characteristic of linear rotation splittings. It is part of the more general modulation of the linear splittings with frequency (seen in Fig. 3, bottom panel). It has the same origin as the similar modulation seen for mode inertia [9] that is due to the trapping of modes (see [20, 21]). The ' $\mathrm{V}$ ' pattern can then constitute a strong indication that the selection of the multiplets is done correctly in observed spectra. In Fig. 3 (middle panel), the integrals expressed in Eq. 1 are plotted, with the kernels computed with the eigenfunctions of each component of the triplet. For the $\mathrm{m}=0$ modes, it is equal to the splitting in the first order formalism. Fig. 3 (middle panel) confirms that modes which belong to the same original multiplet (circled in black) lie in different apparent multiplets, and show quite different p-g nature. Figure 3 (bottom panel) compares the original splittings -i.e. half the differences between frequencies of modes from the same original triplet with opposite $m$ - with the splittings given by the first order approach. The g-m modes (e.g. at $277 \mu \mathrm{Hz}$ and $303 \mu \mathrm{Hz}$ ) have the largest splittings. For such modes, both computations give very close results (with a discrepancy of $\sim 4 \%$ ). The p-m modes (e.g. at $290 \mu \mathrm{Hz}$ and $315 \mu \mathrm{Hz}$ ) have the smallest splittings and show the expected ' $\mathrm{V}$ '-shape variation. For these modes, the discrepancies can reach $30 \%$. This is due to the trapping of these modes which differs from one $m$ to the other. Note that we have also computed original splittings including six spherical harmonics of consecutive angular degree in the non-perturbative calculations and the discrepancies with computations using two $Y_{\ell}^{m}$ are lower than $10^{-3} \%$.

Therefore, between 8 and $20 \mu \mathrm{Hz}$, the fact that the multiplets are correctly selected can be assumed if the splitting given by apparent triplets do follow the ' $\mathrm{V}$ ' pattern. If so, the first order approach provides a correct order of magnitude of the core rotation with the g-m mode splittings, but non-perturbative modeling is required for quantitative conclusions on the rotational profile.

\section{RAPID CORE ROTATING RED GIANTS}

Above $20 \mu \mathrm{Hz}$ (Fig. 1), the mode frequencies no longer behave linearly with respect to rotation. This is due not only to higher order effects that come into play, but also to the trapping of modes that is modified 
Ageing Low Mass Stars: From Red Giants to White Dwarfs

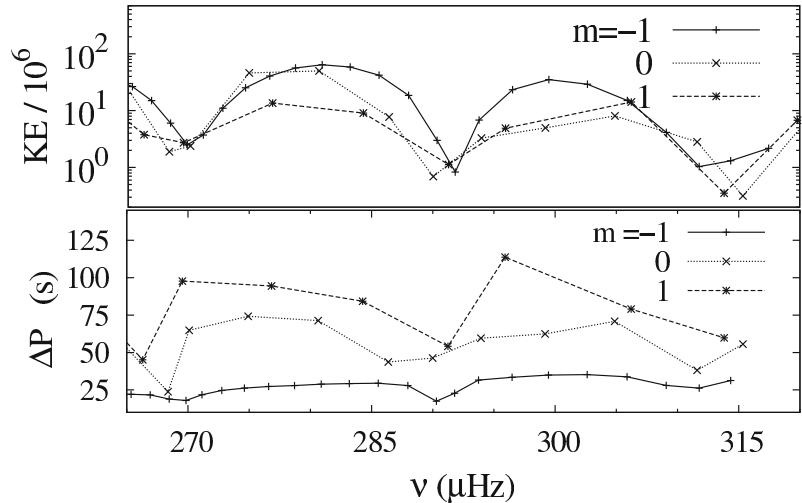

Figure 4. For a model centrally rotating at $180 \mu \mathrm{Hz}$, top: pulsation modes' kinetic energy with respect to their frequency. Bottom: period spacing with respect to the pulsation frequency.

by rotation. Modes which belong to the same original multiplet do not have the same radial distribution. Under these circumstances, the notion of rotational splitting as defined by $\Delta_{n, \ell, m}$ is no longer relevant, and cannot be simply related to the rotation profile.

For instance, for a model with $\Omega_{c} / 2 \pi=60 \mu \mathrm{Hz}$, the first order method gives a splitting of $20 \mu \mathrm{Hz}$ in average. Even worse, when selecting closest modes of opposite $m$ in the synthetic spectra-computed by the complete approach-, the spacing obtained roughly reaches $1 \mu \mathrm{Hz}$. Figure 4 (top) presents the kinetic energy of pulsating modes for a core rotation of $180 \mu \mathrm{Hz}$, which is the rotation obtained by the model of evolution. The large separation, as measured by the difference between consecutive p$\mathrm{m}$ modes with minimum kinetic energies, is conserved. The higher kinetic energy of prograde modes indicates that they have a stronger $g$ nature than retrograde or axi-symmetric ones. This is due to the shift in frequency induced by rotation that brings, in the same frequency range, modes which belong to very different part of the zero rotation spectrum (see Fig. 1).

Figure 4 (top) shows that, in the same range of frequency, the $m=-1$ modes are more numerous than the $m=0$ ones, which, in turn, are more numerous than the $m=+1$ ones. In order to highlight this difference of distribution, the period spacings relative to each $m$ values are plotted in Fig. 4 (bottom). In this diagram, the sub-spectra relative to different azimuthal orders clearly show different values of period spacings, ranging from the lowest for the prograde modes to the highest for the retrograde ones. Note that this phenomenon appears only for rotation high enough that the distributions of the three families of modes associated to $m$ become clearly different $\left(\Omega_{c} \sim 100 \mu \mathrm{Hz}\right.$ for model $\left.\mathrm{M}_{1}\right)$. If one is able to measure three different values of period spacings in a observed spectrum, one is able to identify the values of the azimuthal order. Moreover, based on our study of synthetic spectra, we find that the differences between the values of period spacings increase with $\Omega_{c}$, and therefore can constitute a powerful constraint on the rotation profile.

\section{DISCUSSIONS AND CONCLUSIONS}

Among the observed spectra of red giant stars, some show structures with nearly symmetric spacings around axisymmetric modes. For this kind of spectra, we found that one can figure out if these apparent multiplets split by rotation correspond to the original splittings by plotting the apparent splittings as a function of the axi-symmetric modes frequencies. If the resulting curve follows the ' $\mathrm{V}$ ' pattern, then it is reasonable to state that the triplets are correctly selected, and quantitative information on rotation can be derived from the observed splittings. This corresponds to a low rotation regime when the splitting is smaller than the frequency spacing between two consecutive modes of same $m$, i.e. for $\Omega_{c} /(2 \pi) / \Delta P / P^{2} \sim 2$. If the apparent splittings do not follow the ' $\mathrm{V}$ ' pattern, it means that the triplets overlap. The star is therefore located in the regime of moderate rotation. The frequency differences between the original triplet components are large enough that the trapping differs from one component 
to another. However, if it is still possible to select correctly the original triplets (as done in [6]), the first order approach could be used for a first guess of the core rotation, but non-perturbative modeling is required for a precise determination, particularly because of avoided crossings undergone by $\mathrm{p}-\mathrm{m}$ modes. This rotation regime corresponds to values $\Omega_{c} /(2 \pi) / \Delta P / P^{2}$ between 2 and 5 . Finally, among the observed red giants, some spectra do not show any regular or close to regular structures. We expect these spectra to correspond to rapid rotators. In this case, modes of different azimuthal orders are of very different nature. The concept of rotational splitting of modes with the same radial distribution and different $m$ is no longer relevant, and one should consider separately sub-spectra associated to each values of $m$. Provided that the rotation is high enough to give rise to clearly different distributions with respect to $m$, a new seismic diagnostic can be found by exploiting the differences of period spacings associated to each $m$. That corresponds to the very rapid rotation case i.e. for $\Omega_{c} /(2 \pi) / \Delta P / P^{2} \gtrsim 20$. An intermediate case remains, where the rotational splitting is no longer a relevant seismic diagnostic, but where the rotation is too slow to allow to distinguish three different period spacings associated to the $m$ families of modes (for $\mathrm{X}_{\mathrm{c}} /(2 \mathrm{p}) \times \mathrm{DP} / \mathrm{P}^{2}$ between 5 and 20). Establishing a proper diagnostic in this regime constitutes an important challenge which needs to be addressed in a forthcoming paper.

To sum up, the major impact of moderate rotation on red giants spectra is the modification of the trapping that depends on the azimuthal order. In such a case, the first order perturbative approach gives inaccurate results and the sub-spectra associated to different $m$ carry a different information on the stellar interior. Only methods which compute these $m$-sub-spectra independently, i.e. non-perturbative methods, are appropriate for studying the moderate to rapidly core rotating red giants.

\section{References}

[1] R.M. Ouazzani, M.A. Dupret, D.R. Reese, Astron. Astrophys. 547, A75 (2012), 1209.5621

[2] A. Baglin, M. Auvergne, P. Barge, M. Deleuil, C. Catala, E. Michel, W. Weiss, COROT Team, Scientific Objectives for a Minisat: CoRoT, in ESA Special Publication, edited by M. Fridlund, A. Baglin, J. Lochard, L. Conroy (2006), Vol. 1306 of ESA Special Publication, p. 33

[3] W.J. Borucki, D. Koch, G. Basri, N. Batalha, T. Brown, D. Caldwell, J. Caldwell, J. ChristensenDalsgaard, W.D. Cochran, E. DeVore et al., Science 327, 977 (2010)

[4] S. Deheuvels, R.A. García, W.J. Chaplin, S. Basu, H.M. Antia, T. Appourchaux, O. Benomar, G.R. Davies, Y. Elsworth, L. Gizon et al., Astrophys. Journal 756, 19 (2012), 1206 . 3312

[5] P.G. Beck, J. Montalban, T. Kallinger, J. De Ridder, C. Aerts, R.A. García, S. Hekker, M.A. Dupret, B. Mosser, P. Eggenberger et al., Nature 481, 55 (2012), 1112. 2825

[6] B. Mosser, M.J. Goupil, K. Belkacem, J.P. Marques, P.G. Beck, S. Bloemen, J. De Ridder, C. Barban, S. Deheuvels, Y. Elsworth et al., ArXiv e-prints (2012), 1209.3336

[7] B. Mosser, M.J. Goupil, K. Belkacem, J.P. Marques, P.G. Beck, S. Bloemen, J. De Ridder, C. Barban, S. Deheuvels, Y. Elsworth et al., ArXiv e-prints (2012), 1209.3336

[8] P. Eggenberger, J. Montalbán, A. Miglio, Astron. Astrophys. 544, L4 (2012), 1207.1023

[9] M.J. Goupil, B. Mosser, J.P. Marques, R.M. Ouazzani, K. Belkacem, Y. Lebreton, R. Samadi, ArXiv e-prints (2012), 1211.1546

[10] J. Zahn, Astron. Astrophys. 265, 115 (1992)

[11] J.P. Marques, M.J. Goupil, Y. Lebreton, S. Talon, A. Palacios, K. Belkacem, R.M. Ouazzani, B. Mosser, A. Moya, P. Morel et al., ArXiv e-prints (2012), 1211.1271

[12] H. Kjeldsen, T.R. Bedding, Astron. Astrophys. 293, 87 (1995), arXiv : astro-ph/9403015

[13] W. Unno, Y. Osaki, H. Ando, H. Saio, H. Shibahashi, Nonradial oscillations of stars (1989)

[14] T.G. Cowling, MNRAS 101, 367 (1941)

[15] J. Ballot, F. Lignières, D.R. Reese, M. Rieutord, Astron. Astrophys. 518, A30+ (2010), 1005.0275

[16] T.G. Cowling, R.A. Newing, Astrophys. Journal 109, 149 (1949)

[17] P. Ledoux, Memoires of the Societe Royale des Sciences de Liege 9, 3 (1949) 
Ageing Low Mass Stars: From Red Giants to White Dwarfs

[18] M.j. Goupil, ArXiv e-prints (2011), 1102.1884

[19] D. Lynden-Bell, J.P. Ostriker, MNRAS 136, 293 (1967)

[20] W.A. Dziembowski, D.O. Gough, G. Houdek, R. Sienkiewicz, MNRAS 328, 601 (2001), arXiv: astro-ph/0108337

[21] M.A. Dupret, K. Belkacem, R. Samadi, J. Montalban, O. Moreira, A. Miglio, M. Godart, P. Ventura, H.G. Ludwig, A. Grigahcène et al., Astron. Astrophys. 506, 57 (2009), 0906.3951 